\title{
Bioanalysis Rising Star Award 2018: announcing our finalists!
}

\author{
Buyun Chen, Lisa O'Callaghan \& Jing Tu \\ *Author for correspondence: r.finnie@future-science.com
}

Accepted for publication: 5 June 2018; Published online: 22 August 2018

Over the past 8 years, Bioanalysis and Bioanalysis Zone have been proud to host the Bioanalysis Rising Star Award (formerly the New Investigator Award), to recognize and showcase the most promising early career scientists in our community. The time has now come for you to select your winner for the Bioanalysis Rising Star Award 2018.

We are delighted to present our judges' selection of finalists (in alphabetical order):

- Buyun Chen (Genentech, CA, USA)

- Lisa O'Callaghan (Merck, PA, USA)

- Jing Tu (PPD, VA, USA)

To view Buyun, Lisa and Jing's full profiles and short interviews, please visit www.bioanalysis-zone.com. The 2018 Award is being held in association with Waters Corporation (MA, USA), with the winner receiving a US $\$ 1000$ prize and a year's subscription to Bioanalysis. The winner will be announced and presented with the award at the European Bioanalysis Forum (EBF) open meeting taking place in Barcelona (Spain) in November this year, where they will also present their work.

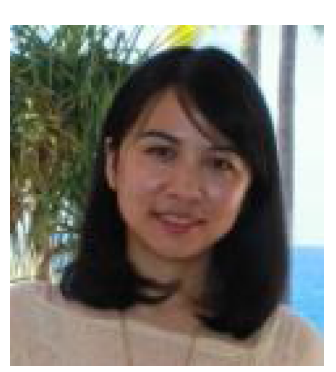

\section{Buyun Chen chen.buyun@gene.com Supporting comments:}

I have been working closely with Buyun Chen for almost 4 years as her supervisor at Genentech. She is a highly motivated scientist and an active contributor to the bioanalytical community. In the past several years, she evaluated and implemented multiple new technologies to improve and extend the capability of the bioanalytical group. One of the examples is that she is the first person to launch the transporter quantitation platform in DMPK department. The quantitation of drug transporter is considered a critical step in in vitro to in vivo extrapolations, but quantitation assays were limited by the absence of pure protein standard and various extraction and digestion conditions. She was able to collaborate with multiple functions and design a rigorous and systematic approach to optimize the conditions and finally setup the robust assay for the quantitation and resulted in successful characterization of multiple in vitro and in vivo research tools used by the company. Overall, she is a creative and collaborative scientist and I am confident in her potential for success. I strongly support her for the Bioanalysis Rising Star Award 2018.

Nominated by:

Alan Deng, Genentech, 1 DNA Way, South San Francisco, CA, USA; deng.yuzhong_alan@gene.com

Q Describe the main highlights of your bioanalytical work?

For my doctoral research, I developed an ionization efficiency enhancement system for oligonucleotide therapeutics that could improve the bioanalytical assay sensitivity by 100 -fold. I also simplified the biological sample extraction procedure for oligonucleotide by using one-step mixed mode solid phase extraction or liquid extraction followed by ethanol precipitation, which significantly reduced the process time (by over $50 \%$ ) and enhanced extraction recovery. These mechanistic research and application studies resulted in several key publications [1-4]. 
At Genentech, I spearheaded the effort to quantify drug transporters to support in vitro and in vivo research model characterization and PBPK modeling. I established a peptide selection procedure for accurate quantitation of drug transporters in both 'absolute' and 'relative' senses. By employing such strategy, I found the hepatic abundance of MDR1 transporter had been underestimated for over 10 times compared with previously reported data [4].

I am currently working on expanding the transporter quantitation platform to more transporters to enhance our understanding of quantitation of endogenous membrane proteins, such as distinguishing active form from inactive form or isolating proteins only from the functional locations.

\section{Q How has your work impacted your laboratory, the bioanalytical field \& beyond?}

After I started the research direction of enhancing oligonucleotide chromatographic separation and ionization efficiency by studying their analytical behavior with different combinations of ion-pairing agents, my lab and at least three other labs from different countries, continued this type of research with both more depth and breadth. My publications on bioanalysis of oligonucleotides remain to be the most cited papers in this field since 2010. Due to the impact of the work, my publication in Journal of Chromatography $A$ was selected as the outstanding manuscript award of AAPS-APQ section in 2013.

My work on transporter quantitation also gained attention in ADME community. I am invited to present my work at Asian Pacific ISSX this year. As a member of the transporter focus group of Simcyp PBPK software, I will be contributing transporter abundance data into the software database together with leading scientists from other major pharmaceutical companies.

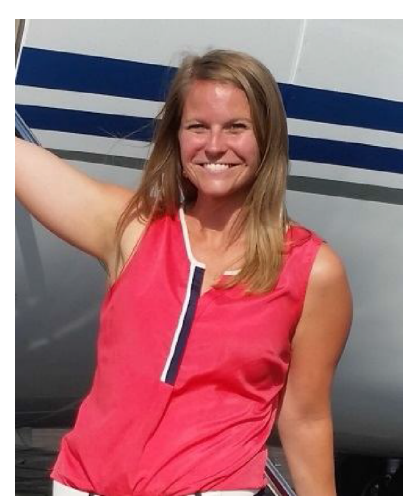

\section{Lisa O'Callaghan} lisa.ocallaghan@merck.com Supporting comments:

Lisa O'Callaghan joined the Pharmacokinetics, Pharmacodynamics and Drug Metabolism department in Merck Research Laboratories (NJ, USA) in 2015 as a Senior Scientist. At Merck, she has focused on the development and implementation of methodology and automation for quantitation of therapeutic monoclonal antibodies in various matrices for preclinical pharmacokinetic (PK) studies. She quickly demonstrated herself to be creative, tenacious and a dedicated scientist. She is a deep technical expert in the area of protein MS. She has used this to effectively establish a strong base for pushing innovation and building a reputation as a forward thinking subject matter expert in the area of protein bioanalysis. She was quickly promoted to Associate Principal Scientist and has transitioned to the role of scientific lead for the protein bioanalysis group within discovery bioanalytics, responsible for the development and application of LC-MS to support biopharmaceutical PK studies and biomarker analysis.

She has been a critical contributor to the establishment of large molecule bioanalytical capabilities within Pharmacokinetics, Pharmacodynamics and Drug Metabolism and Merck \& Co. Her focus has currently turned toward establishing approaches for intact protein quantitation and she leads multiple collaborations for Merck in this space with Agilent Technologies, Purdue University and Q2 solutions. This work has led to significant internal and external presentations and multiple publications, including oral presentations at ASMS (IN, USA, 4-8 June 2017) in 2017 and 2018 (CA, USA, 3-7 June 2018). She is truly a rising star in the field of bioanalysis and the impact of her work will be felt across the field while already influencing the way protein bioanalysis is conducted within Merck and across the biopharmaceutical industry.

Nominated by:

Daniel Spellman, Merck, 770 Sumneytown Pike, West Point, PA, USA; daniel_spellman@merck.com

\section{Q Describe the main highlights of your bioanalytical work?}

The main focus of my work has been examining some of the key questions around the surrogate peptide approach to protein quantitation with LC-MS/MS. The surrogate peptide approach involves an enzymatic digestion of the intact protein into smaller, more LC-MS compatible peptide pieces. Multiple reaction monitoring is used to quantify these surrogate peptides and subsequently infer the parent protein concentration. However, due to the digestion and averaging of heterogeneity that may exist at the protein level, questions remain for this type of assay 
regarding what population (or mix of protein forms) is being represented. I have been working on developing methods to alleviate some of these concerns through a sequential immunoaffinity purification that should ensure a single population of protein is being quantitated. In addition, we have looked to eliminate the digestion step completely, pursuing the application of intact protein quantitation with high resolution MS. I have conducted an exhaustive evaluation of sample preparation, protein separation, intact protein MS, and data analysis approaches. We have made great strides in demonstrating that practical utility of such an approach, eliminating the digestion step completely and increasing sample throughput.

\section{Q How has your work impacted your laboratory, the bioanalytical field \& beyond?}

The work I have done around intact monoclonal antibody quantitation has been a hot topic at many of the recent conferences I have attended. As the resolution and sensitivity of high resolution MS instrumentation has increased, the possibility of intact quantitation has begun to be realized across many of the top pharmaceutical companies. Through this work, I have built several networks with groups working on similar applications at other top Pharma companies. We have had a continuously open dialog about sample preparation techniques, methods and current limitations. Most recently, I was invited to participate in an oral session at Clinical \& Pharmaceutical Solutions through Analysis (CPSA)-centered specifically on intact quantitation in PK. This session included speakers from Janssen (Beerse, Belgium) and GlaxoSmithKline (Brentford, UK) in addition to myself, all discussing our most recent efforts to improve intact quantitation.

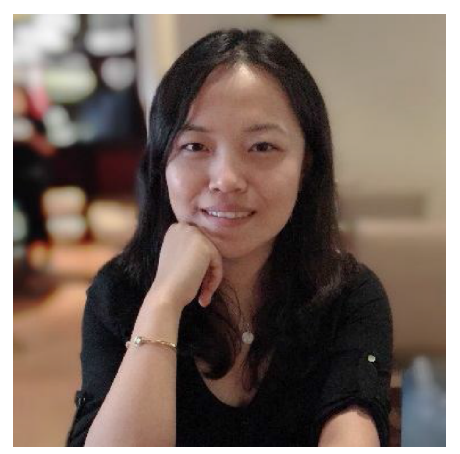

\section{Jing Tu \\ jing.tu@ppdi.com \\ Supporting comments:}

Jing has consistently shown an extremely high level of leadership and strong technical capabilities since joining PPD $^{\circledR}$ Laboratories. She has led the integration of new technologies and implemented a systematic ligand-binding assay (LBA) biomarker method development strategy that significantly improved quality, method consistency and method development efficiency. During her time at PPD, she has been developed a growing group of scientists. Her team members have given presentations at international conferences and participated in the bioanalytical community. She has also been heavily involved in PPD's cross-lab, cross-functional strategy development. Because of her key contributions, she was awarded a PPD-CEO performance excellence award in 2016.

She serves as a committee member of the Global CRO Consortium, the organizing committee for the APA meeting and as a member of the AAPS bioanalytical terminology team. She has given presentations at EBF, AAPS, WRIB, SQA (Society of Quality Assurance), CPSA and APA conferences regarding biomarker bioanalytical related challenges.

In short, she has made multiple scientific contributions that have had a significant impact on the field of LBA bioanalysis and the PPD Laboratories biomarker lab. She is a tremendous resource for our company and bioanalytical community. I strongly and enthusiastically support her in the Bioanalysis Rising Star Award competition 2018.

\section{Nominated by:}

Patrick Bennett, PPD, 2244 Dabney Road, Richmond, VA, USA; Patrick.Bennett@ppdi.com

\section{Q Describe the main highlights of your bioanalytical work?}

During my first bioanalytical job, I had the opportunity to develop and validate 11 LBA under US FDA guidelines and industry white papers. These methods have been used by well-established national and international pharmaceutical companies to support their preclinical and clinical PK, pharmacodynamics and anti-drug antibody evaluations.

In my current role, I started a team that is dedicated to biomarker LBA method development and validation/qualification. Important initiatives I have led include the integration of new technologies (i.e., ELLA and Quanterix) into our laboratories and the implementation of a systematic LBA biomarker method development strategy. My team's goal is to develop customized solutions for our clients that enable a context-of-use, fit-for- 
purpose approach to biomarker analysis, adapting good clinical laboratory practice/good laboratory practice or College of American Pathologists/Clinical Laboratory Improvement Amendments, as appropriate.

Thus far in my career, I have published two peer-reviewed papers, introduced six posters and given five presentations at international conferences, such as EBF, CPSA and SQA.

\section{Q How has your work impacted your laboratory, the bioanalytical field \& beyond?}

Traditionally, matrix effects, minimum required dilution, dilutional linearity, selectivity and sensitivity are evaluated from different experiments for bioanalytical methods. Over the last decade, many discussions have been published in scientific literature regarding the conduct of parallelism experiments, but the experiment has not been considered as a critical experiment for traditional PK/PD method development and validation.

I proposed a systematic approach that can be applied to guide endogenous analyte LBA method development and optimization with a suggested way to interpret parallelism data. This approach has been implemented in my company's laboratories, which reduced method development time to 5 days with critical reagents available. Similar ideas also are applicable to PK LBA method development using a 'spiked parallelism' approach.

As previously noted, I have published papers and posters, as well as given talks and exchanged ideas with industry experts, to contribute to the bioanalytical community and beyond.

\section{Financial \& competing interests disclosure}

The authors have no relevant affiliations or financial involvement with any organization or entity with a financial interest in or financial conflict with the subject matter or materials discussed in the manuscript. This includes employment, consultancies, honoraria, stock ownership or options, expert testimony, grants or patents received or pending, or royalties.

No writing assistance was utilized in the production of this manuscript.

\section{References}

1. Chen B, Mason SF, Bartlett MG. The effect of organic modifiers on electrospray ionization charge-state distribution and desorption efficiency for oligonucleotides. J. Am. Soc. Mass. Spectrom. 24(2), 257-264 (2013).

2. Chen B, Bartlett MG. Evaluation of mobile phase composition for enhancing sensitivity of targeted quantification of oligonucleotides using ultra-high performance liquid chromatography and mass spectrometry: application to phosphorothioate deoxyribonucleic acid. $J$. Chromatogr. A 1288, 73-81 (2013).

3. Chen B, Bartlett M. A one-step solid phase extraction method for bioanalysis of a phosphorothioate oligonucleotide and its 3' n-1 metabolite from rat plasma by uHPLC-MS/MS. AAPS J. 14(4), 772-780 (2012).

4. Chen B, Liu L, Ho H et al. Strategies of drug transporter quantitation by LC-MS: importance of peptide selection and digestion efficiency. AAPS J. 19(5), 1469-1478 (2017). 